Research Article

\title{
Effects of Contact Angle on the Hysteresis Effect of Soil-Water Characteristic Curves during Dry-Wet Cycles
}

\author{
Gaoliang Tao $\mathbb{D}^{1,2}$ Ziyue Li, ${ }^{1}$ Lisheng Liu, ${ }^{2}$ Yangyang Chen, ${ }^{1}$ and Kai Gu ${ }^{1}$ \\ ${ }^{1}$ School of Civil Engineering, Architecture and Environment, Hubei University of Technology, Wuhan, Hubei 430068, China \\ ${ }^{2}$ School of Urban Construction, Wuchang University of Technology, Wuhan, Hubei 430223, China \\ Correspondence should be addressed to Gaoliang Tao; tgl1979@126.com
}

Received 3 October 2020; Revised 26 March 2021; Accepted 1 April 2021; Published 12 April 2021

Academic Editor: Musa Adamu

Copyright (C) 2021 Gaoliang Tao et al. This is an open access article distributed under the Creative Commons Attribution License, which permits unrestricted use, distribution, and reproduction in any medium, provided the original work is properly cited.

The hysteresis characteristics of soil-water characteristic curves (SWCCs) under dry-wet cycling conditions are very important for understanding unsaturated soil properties, so it is crucial to propose an accurate and efficient method for predicting the hysteretic behaviors of SWCCs. To this end, this paper investigates the hysteresis characteristics of SWCCs in the full suction range of seven kinds of Hunan red clay with different initial dry densities by combination of the pressure plate method, the paper filter method, and the saturated salt solution method. It is found that there are, respectively, strong and weak hysteresis zones in the drying and wetting SWCCs under dry-wet cycling conditions. By combining this feature and based on the drying curve, the soil volume and contact angle changes during the drying and wetting processes are employed to predict the hysteretic behaviors of SWCCs. To verify the validity of the prediction method, the predicted curves of the samples with different initial dry densities are compared with the measured curves. The results show that in the strong hysteresis zone, the hysteresis characteristics of the drying and wetting SWCCs are mainly resulted from the changes in the soil pore structure; in the weak hysteresis zone, the hysteresis characteristics are mainly influenced by the changes in the receding and advancing contact angles corresponding to the drying and wetting processes. The Young-Laplace theory is used to transform the changes of contact angle during the drying and wetting processes into the proportional relationship $k$ of matric suction, and the corresponding wetting curve is obtained by smoothing the drying curve. It is found that the prediction effect in the high suction part (the strong hysteresis zone) is better than that in the weak hysteresis zone, which confirms that the hysteresis effect of SWCCs in the high suction part is influenced by the contact angle. Our proposed method can greatly reduce the test period and has a significant practical application value, which provides a new idea for the prediction of SWCCs under dry-wet cycling conditions.

\section{Introduction}

The soil-water characteristic curve (SWCC) describes the relationship between matric suction and water content (saturation) and can be used as an indirect index for understanding the shear strength [1], permeability, and deformation characteristics of the soil [2]. The SWCC measurement methods mainly include the pressure plate method, the filter paper method, the salt solution method, and the TDR matric suction measurement method (a method of measuring the matric suction by a suction probe, which is applicable to the state of $0-300 \mathrm{kPa}$ ). The direct measurement is a method that uses equipment (e.g., pressure plate and tensiometer) to measure the water content of the soil under a certain matric suction. This method can only measure SWCCs in a specific state (the same dry density or void ratio). Also, it is difficult to use a single method to measure SWCCs in the full suction range. On account of this, a combination of several methods is required to more effectively reflect the relationship between the soil-water content during dry-wet cycles and the matric suction. Among them, the hysteresis phenomenon reflected in this process has attracted extensive attention from a majority of geotechnical workers. Some scholars tend to use mathematical models for the prediction or mathematical representation of SWCCs. In the existing literature, equations of soil-water characteristic curves, such as Gardner [3], Brooks-Corey [4], Van Genuchten [5], and Fredlund-Xing 
[6], are usually used to fit experimental data to derive the water content as a function of matric suction. However, this method does not consider the hysteresis phenomenon of the soil-water characteristic curve during the dry-wet cycles of the soil. Since the 1960s, many scholars have made great efforts and proposed various models for predicting the hysteresis characteristics of SWCCs, including the empirical model [7-9], the domain model [10-14], the boundary model [15-18], the rational extrapolation model [19], and the hysteresis simulation method [20-23]. In these models, the domain model has perfect theoretical foundation, and its prediction results are fairly accurate. Nevertheless, the calculation process of this model is very complicated, and it is inconvenient to use the model for predicting the hysteresis characteristics of SWCCs [18].

Under dry-wet cycling conditions, there are many factors that affect the hysteresis effect of SWCCs, such as the ink bottle effect [24], the contact angle hysteresis, the pore size unevenness, the initial dry density, the number of wet and dry cycles, the stress, and the particle size. $\mathrm{Ng}$ et al. [25] analyzed the effects on the SWCCs of Hong Kong volcanic ash soils in terms of initial dry density, initial water content, dry and wet history, soil structure, and stress, and the results indicated that the SWCCs of remodeled samples were very different from those of natural samples. He et al. [26] analyzed the reasons for the hysteresis effect of unsaturated soil-water characteristic curves during the dry-wet cycles, and their results showed that the difference between the contact angles of the wetting surface and the drying surface is the main cause from a microscopic perspective. To better describe the strength of the hysteresis characteristics of SWCCs, Zhang et al. [27] proposed a concept of "hysteresis degree" to investigate the hysteresis characteristics of SWCCs. Li et al. [28] calculated the hysteresis values of the soil by changing the initial dry density, the number of drywet cycles, and the vertical stress and attributed the hysteresis properties of SWCCs to the change in pore volume. The hysteresis value is the ratio of the maximum difference between the volumetric water content on the drying curve and the wetting curve and the difference between the corresponding saturated volumetric water content and the residual volumetric water content during the dry-wet cycles. $\mathrm{Wu}$ et al. [29] determined the changes of matric suction of expansive soil under different numbers of dry-wet cycles by the filter paper method and established an SWCC model of expansive soil considering the effects of dry-wet cycles. Unfortunately, those above studies all fail to study SWCCs in the full suction range.

So far, due to the long test period, the high price of test equipment, and prone to test errors and other factors, there have been relatively few reports in terms of investigating the effects of dry-wet cycles on the hysteresis effect of SWCCs in the full suction range, which makes it necessary to spend a lot of human and material resources to obtain a full suction range dry-wet SWCC. Sun et al. [30] conducted a soil-water characteristic test on Nanyang swelling soil in the full suction range. The test results showed that the hysteresis loop formed by the drying and wetting curves in the low suction range was extremely obvious, and that the hysteresis effect of the SWCC produced by the dry-wet cycles disappeared when the suction force is greater than $300 \mathrm{MPa}$. Gao et al. [31] took the pearl soil as the research object and concluded that there were significant hysteresis phenomena in their SWCCs under different initial void ratio conditions. On that basis, they proposed a method for normalizing SWCCs for different initial void ratios in the full suction range. Cai et al. [32] conducted an experimental study on SWCCs in the full suction range for mud soil samples and compacted soil samples, and the results showed that the pore structure has a significant effect on SWCCs.

The hysteresis behaviors of hydraulic characteristics can be attributed to two internal reasons. One is that the pore size of soil particles changes during dry-wet cycles, and the other is that the solid-liquid contact angle can be divided into advancing angle and receding angle on the microscopic scale. This view is consistent with the conclusions of Zheng et al. [33] and He et al. [26]. In this study, the drying and wetting SWCCs of Hunan red clay in the full suction range are obtained by the pressure plate method, the filter paper method, and the saturated salt solution method. In combination with the soil volume change curves of the drying and wetting processes, the Young-Laplace theory is used to estimate the change in contact angle and thus predict the hysteresis effect of SWCCs in the high suction range. With this method, the workload of the test measurement curve can be lowered and the test difficulty can be greatly reduced so that the hysteresis behaviors of SWCCs during dry-wet cycles can be predicted more efficiently. Our work is of great significance to the study on the hydraulic properties of unsaturated soil and makes it more convenient to investigate the engineering properties of unsaturated soil.

\section{Measurement of SWCCs in the Full Suction Range}

2.1. Basic Physical Properties of Test Soil Samples. The main characteristics of this soil are of high clay content and low permeability. Through the compaction test, the optimum water content is determined as $19 \%$. Other basic physical property tests were conducted on the soil samples to obtain the basic physical indexes, as shown in Table 1 .

2.2. Measurement Methods. Although the filter paper method can measure the full range of soil suction, to ensure the testing accuracy, this paper uses a combination of three soil suction measurements (the pressure plate method, the filter paper method, and the saturated salt solution method) to achieve the full suction range measurement of the drying and wetting SWCCs of Hunan red clay with seven different initial dry densities.

For the pressure plate method, the used instrument was from U.S. Newman Company and mainly consisted of pressure gauge, pressure chamber, ceramic plate, nitrogen gas source, and other devices. The implementation process is detailed in [34].

The filter paper method is employed to configure the target water content. For every two groups, the sample has 
TABLE 1: The basic physical properties of Hunan red clay.

\begin{tabular}{lccccc}
\hline Sample & Specific gravity of soil $\left(G_{\mathrm{S}}\right)$ & Liquid limit, $w_{\mathrm{L}}(\%)$ & Plastic limit, $w_{\mathrm{P}}(\%)$ & Plasticity index $\left(I_{\mathrm{P}}\right)$ & $\begin{array}{c}\text { Natural water content, } \\
w(\%)\end{array}$ \\
\hline Hunan red clay & 2.76 & 46.34 & 27.84 & 18.5 & 13.8 \\
\hline
\end{tabular}

the same dry density and water content. There are two samples between three layers of filter paper. The upper and lower layers are the protective filter paper, and the middle layer is the test paper. The size of the protective filter paper is larger than that of the test filter paper. The gap between the samples needs to be sealed with insulating tape. Then, the samples are put into a sealed box, and the box is placed into an incubator. To ensure the testing accuracy, the mass of the filter paper is measured by an electronic balance with an accuracy of $0.0001 \mathrm{~g}$. After the samples are balanced, the sealed box is opened and the filter paper is quickly removed with tweezers for weighing. In measuring the soil-water characteristic curve of the wetting process, we saturate the prepared sample with vacuum, which is achieved with the help of a vacuum pump and a pressure chamber. First, the sample is put into the pressure chamber and pumped by using the vacuum pump to make the air pressure in the chamber $-0.1 \mathrm{MPa}$ for $1 \mathrm{~h}$; then, the sample is filled with water in the pressure chamber until it is submerged and left for $10 \mathrm{~h}$. The saturated sample is placed indoors with a fan until the water content does not change. Then, they are placed in a curing box and humidified evenly to the target water content. After that, the samples are sealed with plastic wrap and the water is left to migrate evenly. The measurement process is consistent with dehumidification (drying). The test filter paper is Whatman No. 203, which has a different suction calibration curve than Whatman 42. In the literature, Wang et al. [35] and Bai et al. [36] have measured the suction calibration curve for Whatman No. 203 filter paper, which is expressed as follows:

$$
\begin{cases}\lg \psi=5.493-0.0767 w_{\mathrm{f}}, & w_{\mathrm{f}} \leq 47 \% \\ \lg \psi=2.470-0.0120 w_{\mathrm{f}}, & w_{\mathrm{f}}>47 \%\end{cases}
$$

where $\psi$ is the suction and $w_{\mathrm{f}}$ is the water content of the filter paper after equilibration.

The saturated salt solution method is used to control the relative humidity [37] $(\mathrm{RH})$ of the pore water vapor by different saturated salt solutions in order to achieve different total suction forces. When the $\mathrm{RH}$ value of water vapor in the pores of the soil sample is less than $100 \%$, it means that there is a suction between the pores of the soil. Hence, the effects of controlling soil suction can be obtained indirectly by varying the $\mathrm{RH}$ values corresponding to different saturated salt solutions. The process is detailed in [38].

\subsection{SWCCs of the Dry-Wet Cycle in the Full Suction Range.} In order to analyze the effect of hysteresis on SWCCs under dry-wet cycling conditions in the full suction range, the pressure plate method, the filter paper method, and the saturated salt solution method are integrated to draw SWCCs with different initial dry densities during the drying and wetting processes. As shown in Figure 1, the results from the three test methods are well related, and the SWCCs of Hunan red clay can be measured effectively within the full suction range. The three test data show the same variation rules; that is, the volume water content decreases with the increase in the matric suction and the wetting curve is located below the drying curve, which is an obvious hysteresis phenomenon. This phenomenon can be attributed to the internal pore structure changes during the process of drying and wetting of the soil. Compared with the drying process, a part of the water content in the internal pore channels of the soil cannot be recovered by wetting. As the matric suction increases, the drying and wetting SWCCs of the samples with different initial dry densities are close to each other, and the hysteresis area enclosed by the drying and wetting SWCCs also decreases. In the strong hysteresis zone, the area of the hysteresis loop is much larger than that in the weak hysteresis zone, and the two have an intuitive dividing line. To further investigate this phenomenon, the effects of contact angle on the hysteresis characteristics of SWCCs during dry-wet cycles are investigated, and the relationship between the contact angle and the hysteresis characteristics of SWCCs is analyzed by measuring the contact angle of Hunan red clay with a contact angle measurement instrument.

\section{Contact Angle Measurement Experiment}

3.1. Contact Angle Definition and Its Hysteresis Principles. For unsaturated soil systems, the contact angle is defined as the angle between a line tangent to the gas-liquid interface and a line formed by the liquid-solid interface [39], as shown in Figure 2. When the surface of the solid is rough, chemically inhomogeneous, or contaminated in some way, the contact angle of the solid surface is not unique as indicated by the Young-Laplace equation (equation (3)) but varies between some minimum and some maximum angles, which is called contact angle hysteresis. The solid-liquid contact angle changes as the soil undergoes a dry-wet cycle, where the dehumidification (drying) process corresponds to the receding angle and the hygroscopic (wetting) process to the advancing angle. When the droplet volume increases, the contact angle is the advancing contact angle when the threephase line of contact between the droplet and the solid surface is about to move but has not moved; when the droplet volume decreases, the contact angle is the receding contact angle when the three-phase line of contact between the solid and the liquid is about to move but has not moved. The advancing and receding angles are defined schematically as shown in Figure 3. The advancing contact angle indicates a decrease in matric suction, and the receding contact angle indicates an increase in matric suction. 


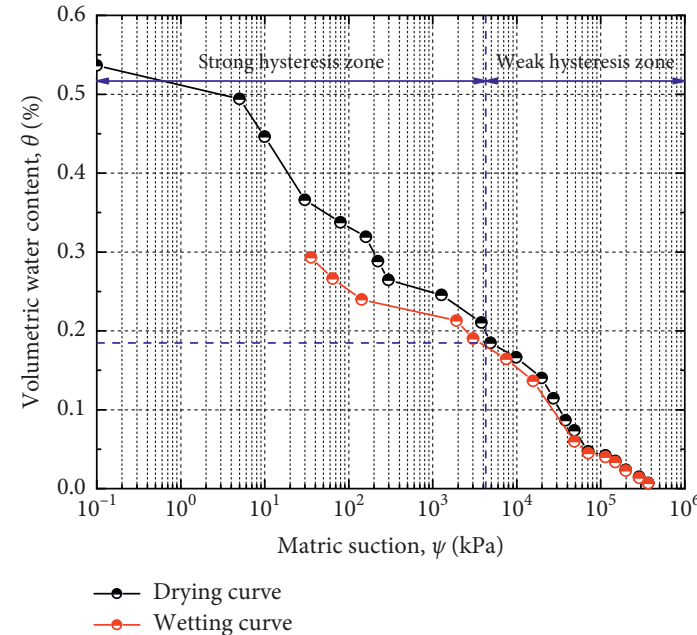

(a)

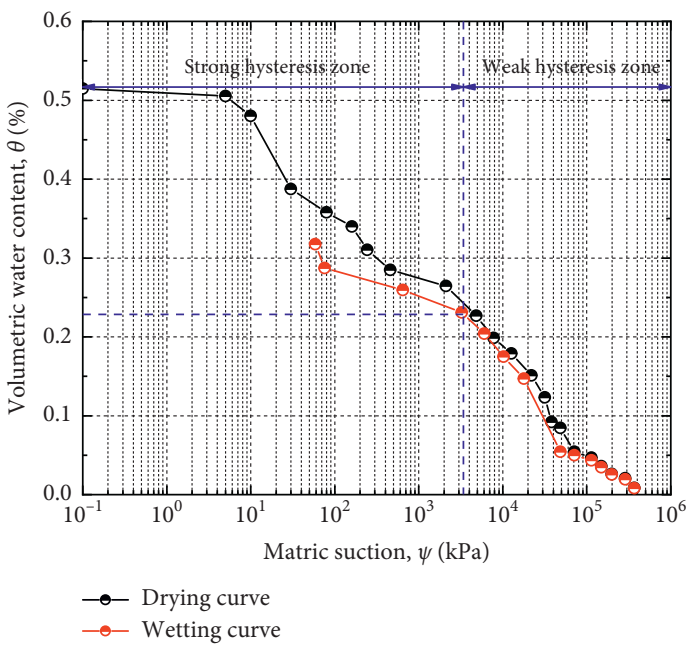

(c)

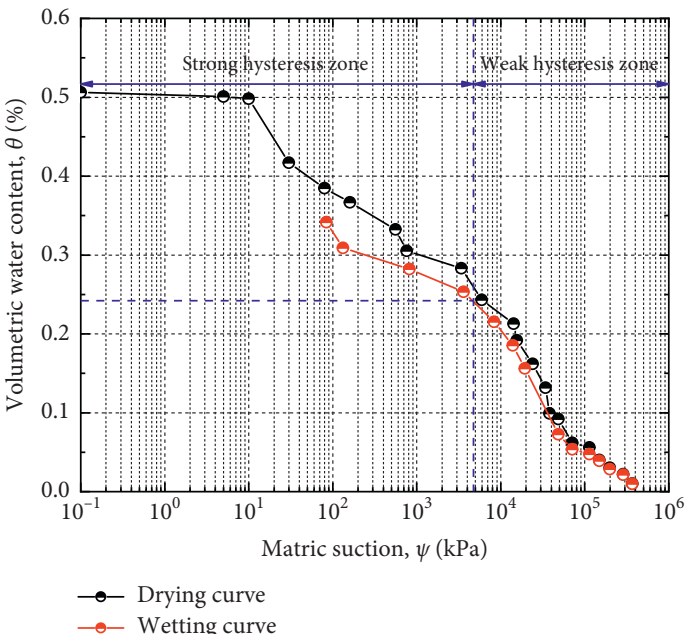

(e)

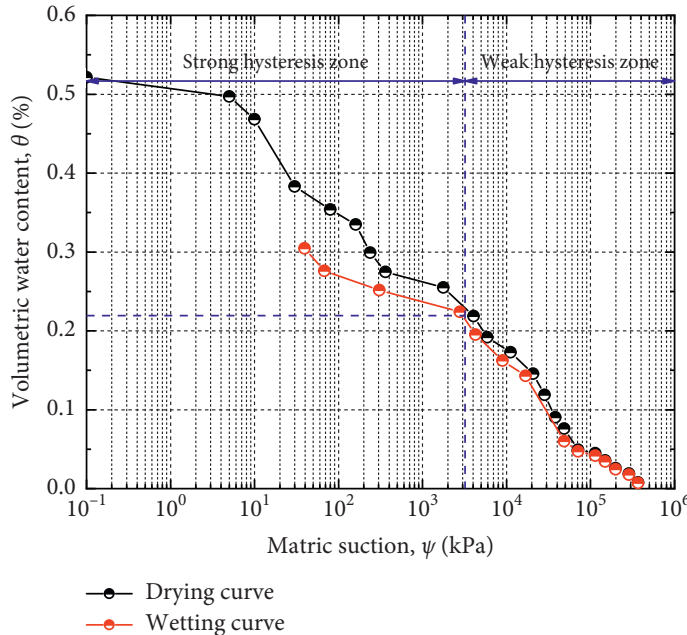

(b)

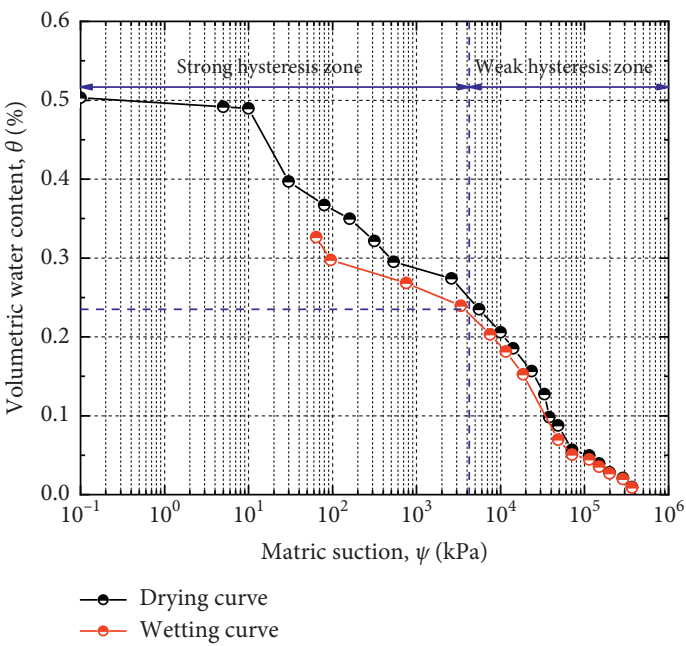

(d)

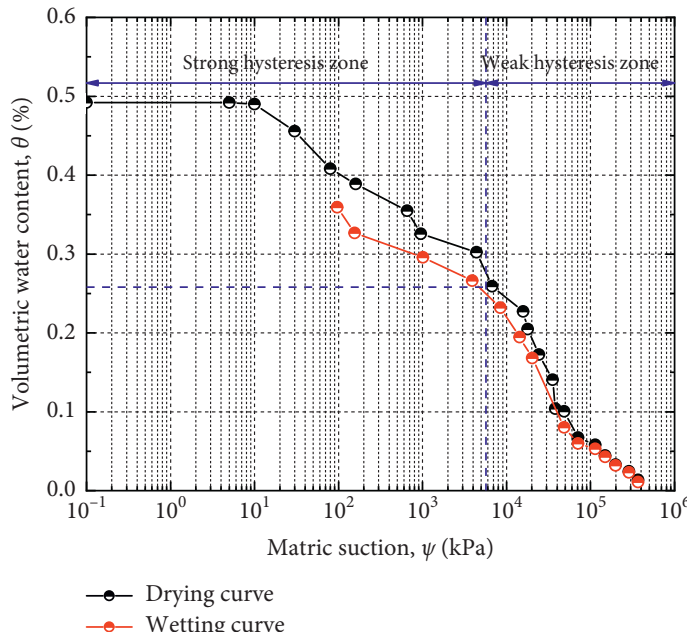

(f)

Figure 1: Continued. 


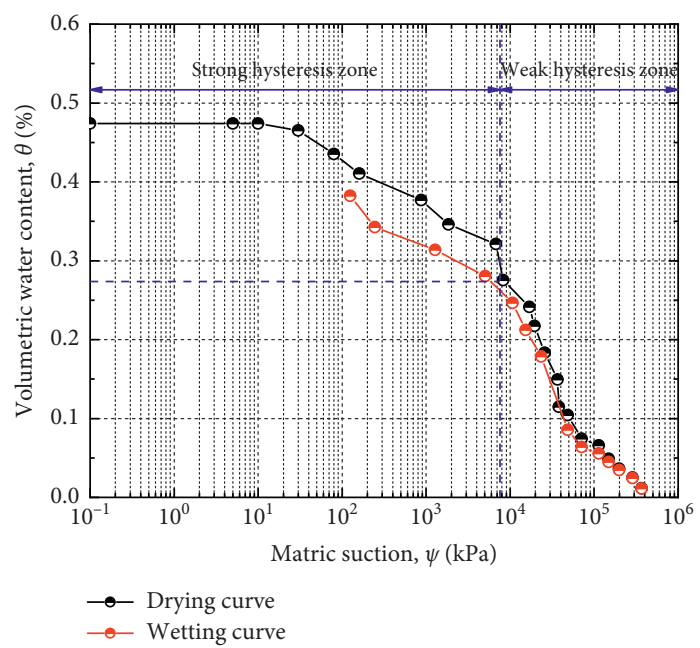

(g)

FIGURE 1: Soil-water characteristic curves of wet and dry cycles with different initial dry densities in the full suction range: $(\mathrm{a}) \rho_{\mathrm{d}}=1.3 \mathrm{~g} / \mathrm{cm}^{3}$; (b) $\rho_{\mathrm{d}}=1.35 \mathrm{~g} / \mathrm{cm}^{3} ;$ (c) $\rho_{\mathrm{d}}=1.4 \mathrm{~g} / \mathrm{cm}^{3} ;$ (d) $\rho_{\mathrm{d}}=1.45 \mathrm{~g} / \mathrm{cm}^{3} ;$ (e) $\rho_{\mathrm{d}}=1.5 \mathrm{~g} / \mathrm{cm}^{3} ;$ (f) $\rho_{\mathrm{d}}=1.6 \mathrm{~g} / \mathrm{cm}^{3} ;(\mathrm{g}) \rho_{\mathrm{d}}=1.7 \mathrm{~g} / \mathrm{cm}^{3}$.

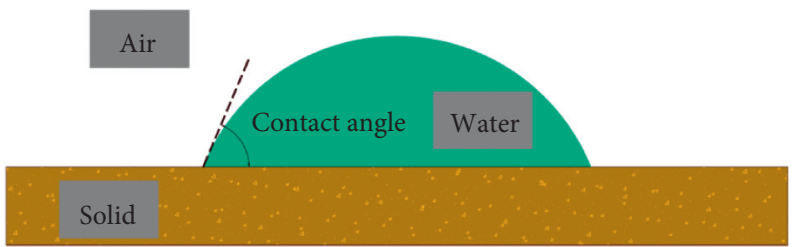

Figure 2: Definition of contact angle.

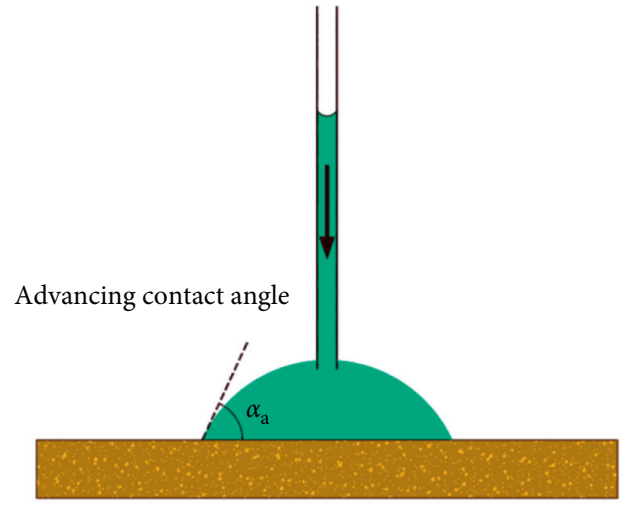

(a)

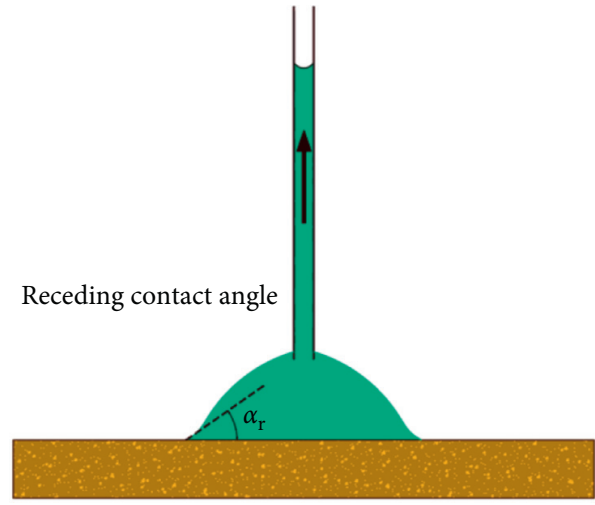

(b)

FIGURE 3: Definition of advancing and receding contact.

3.2. Sample Preparation. The study on three-phase contact angles has matured in materials science and surface physics and chemistry [40], but there is still much room for improvement regarding the measurement of soil contact angles in the field of unsaturated soils. Many methods have been proposed to measure soil contact angles, including the slant plate method, the hanging sheet method, the lying drop method, the suspended bubble method, the Wilhelmy method, the capillary method, and the increasing or decreasing droplet method.
The contact angle measuring equipment used in this study is a German KRUSS-DSA30 contact angle measuring instrument, and the test instrument is shown in Figure 4. The instrument measures the advancing and receding angles by observing the changes in contact angle during the process of increasing or decreasing the droplet.

The sample preparation process of the contact angle test is as follows. The dried, crushed Hunan red clay was sieved through sieve pores of $2 \mathrm{~mm}$ and configured to achieve the optimum water content. Afterwards, the soil 


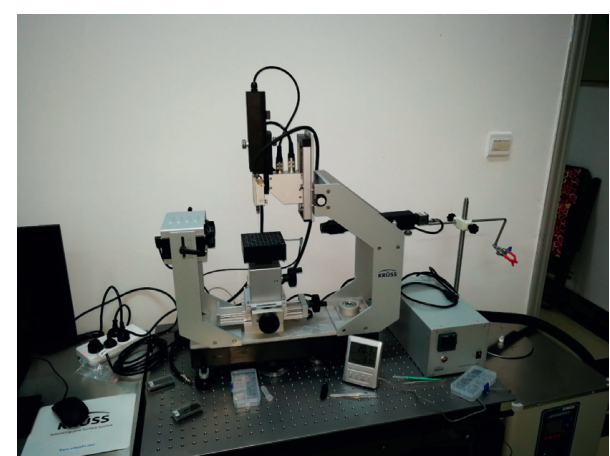

FIGURE 4: KRUSS-DSA30 contact angle measuring instrument.

was placed in a thermostat for three days to allow the water in the soil to migrate evenly. The soil was pressed into thin slices with a thickness of about $2-3 \mathrm{~mm}$ by a hydraulic jack and then cut into squares with the same length and width of $2 \mathrm{~cm}$ by a blade. The main concern of this study is the effect of the contact angle of the soil surface on the hysteresis effect of the soil-water characteristic curve. Because the contact angle test is strongly influenced by the flatness of the sample surface, in this experiment, we used a brush to remove small soil particles from the contact surface to ensure the accuracy of the experimental results.

3.3. Contact Angle Measurement. Before the test begins, the contact angle measurement instrument and the connected computer are turned on in advance for checking potential abnormalities in the associated indicator lights and computer. A syringe containing a certain amount of distilled water is fixed in the slot of the holder above the carrier table and perpendicular to the carrier table. It should be noted that when the table is adjusted up and down, the syringe needle should be kept at a certain distance to prevent it from being bent or damaged.

The contact angle measurement can be carried out according to the following procedure. First, open the ADVANCE software on the desktop and enter the contact angle measurement interface. Then, turn on the camera and adjust the brightness of the light source. After that, place the sample on the slide and put it on the bench. Here, it is noted that the height of the bench should be carefully adjusted so that the surface of the sample can be displayed on the computer. Next, select the droplet content and click the Drop button to drop the droplet on the sample surface. In order to find the angle between the droplet and the baseline of the sample, select the automatic baseline in the software and click Enter button to confirm the selection. Thus, the contact angle can be measured and the data can be recorded by the computer. To ensure the testing accuracy, four groups of squares with the same size should be measured and the minimum value of the receding angle and the maximum value of the advancing angle are taken as the final results. Figures 5(a) and 5(b) show the measurement results of the advancing and receding angles, respectively, and the numerical results are listed in Table 2.

\section{Prediction Results}

4.1. Scale Factor $k$ and Wetting Curves for Contact Angle Changes. Considering the contact angle hysteresis effect, the Young-Laplace theory is introduced to find the proportional relationship between the soil dehumidification (drying) and the matric suction in the process of hygroscopic (wetting) absorption, and the dehumidification (drying) curve is translated to get the SWCC for the initial dry density $\rho_{0}$ if the soil advancing and receding angles are known.

First, the receding angle of the soil dehumidification (drying) process is known as $\alpha_{1}$, and the advancing angle of the hygroscopic (wetting) process is $\alpha_{2}$. Here, $\alpha_{1}<\alpha_{2}$. According to the Young-Laplace theory, the relationship between the matric suction $\psi$ and the effective pore diameter $r$ can be expressed as follows:

$$
\psi=\frac{2 T_{s} \cos \alpha}{r},
$$

where $T_{s}$ is the surface tension, $\alpha$ is the contact angle, and $2 T_{s}$ $\cos \alpha$ is a constant at a certain temperature.

From equation (2), the proportional relationship $k$ for the cosine of the contact angle during drying and wetting processes can be obtained:

$$
k=\frac{\psi_{1}}{\psi_{2}}=\frac{\cos \alpha_{1}}{\cos \alpha_{2}} .
$$

Thus, the above SWCC for the full suction range in the desorption process can be shifted according to the proportional relationship $k$. Then, the final SWCC for the drywet cycles with the initial dry density $\rho_{0}$ can be obtained. In Section 3.3, the dehumidification (drying) receding angle is known as $37.8^{\circ}$ and the advancing angle is $55.4^{\circ}$. Thus, $k$ can be calculated as 1.392 by equation (3). Based on the above measured data of the dehumidification (drying) SWCCs of Hunan red clay in the full suction range for seven groups of samples with different initial dry densities, the matric suction for the corresponding hygroscopic (wetting) process at the same water content can be obtained by multiplying the matric suction during the dehumidification (drying) process with the scale factor $k$. Figure 6 provides the measured and predicted SWCCs during dry-wet cycles in the full suction range.

4.2. Analysis. It can be seen from Figure 6 that, in the interval of $0.1-3 \mathrm{MPa}$, the effects of contact angle on the hysteresis effect of dry-wet cycling SWCCs are not significant; the predicted values are even greatly different from the measured ones. This indicates that the dominated influencing factor for the hysteresis effect of dry-wet cycling SWCCs in this suction interval is not the contact angle. In the suction interval greater than $3 \mathrm{MPa}$, the predicted values are almost the same as the measured ones, indicating that the predicted hygroscopic (wetting) SWCC of this suction range is in better agreement with the measured results. Hence, it can be concluded that the contact angle and the Young-Laplace theory can well predict the hysteresis effect in the high suction part of the SWCC during dry-wet cycles but 


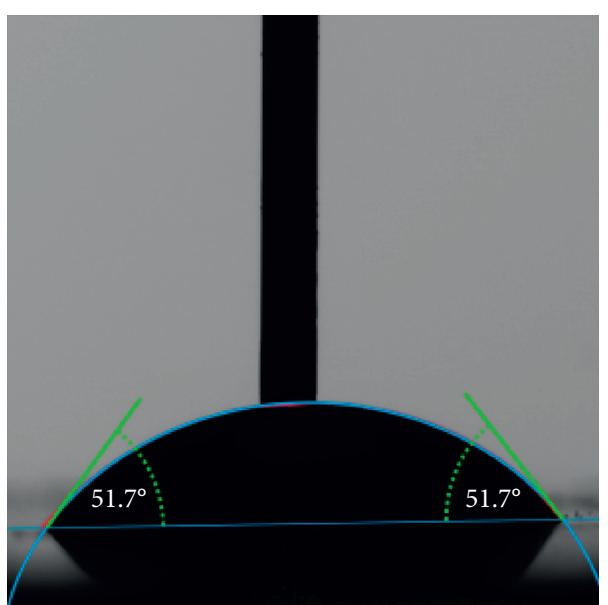

(a)

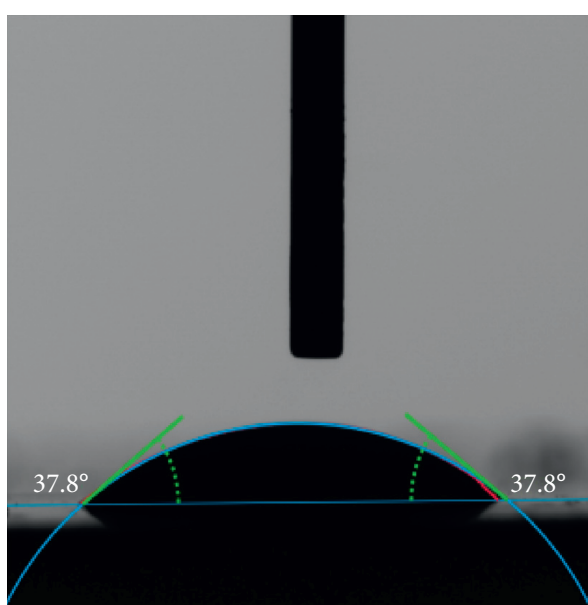

(b)

FIGURE 5: Angle measurement results: (a) advancing angle; (b) receding angle.

TABLE 2: Four groups of samples contact angle measured values.

\begin{tabular}{|c|c|c|c|c|c|}
\hline \multirow{2}{*}{ Type of contact angle } & \multicolumn{4}{|c|}{ Specimen number } & \multirow{2}{*}{ Extreme value } \\
\hline & $\mathrm{S} 1$ & S2 & S3 & S4 & \\
\hline Advancing angle & $53.2^{\circ}$ & $53.9^{\circ}$ & $55.4^{\circ}$ & $51.7^{\circ}$ & $55.4^{\circ}$ \\
\hline Receding angle & $39.7^{\circ}$ & $39.8^{\circ}$ & $40.6^{\circ}$ & $37.8^{\circ}$ & $37.8^{\circ}$ \\
\hline
\end{tabular}

performs poorly in the low suction part. The research on the hysteresis effect of soil-water characteristic curve in the low suction interval is mature, but the research on the hysteresis effect of soil-water characteristic curve in the high suction interval is insufficient. The existence of the hysteresis effect directly leads to the fact that the soil strength of the dehumidification (drying) process is different from that of the hygroscopic (wetting) process of the same water content, which will have a certain impact on the actual project. Therefore, it is necessary to study the hysteresis effect of soilwater characteristic curve under high suction. At the same time, the prediction of the wetting curve in the high suction interval will greatly reduce the workload of determining soilwater characteristic curve in this paper.

\section{Discussion}

As shown in Figure 6, with the gradual increase in the initial dry density, the dividing line between the strong hysteresis zone and the weak hysteresis zone shifts to the right accordingly, i.e., the range of the strong hysteresis zones to expand on the increase in the dry density. However, the boundary values for both the strong and weak hysteresis zones of Hunan red clay are within the suction range of $10^{3}-10^{4} \mathrm{kPa}$. In this section, we discuss the methods used to determine the suction boundary values for the strong and weak hysteresis zones. A sample with an initial dry density of $1.3 \mathrm{~g} / \mathrm{cm}^{3}$ is used as an example to investigate the variation of dry density with water content during the drying and wetting processes. The test procedure is as follows: first, the saturated sample is placed in a constant temperature drying oven for dehumidification (drying), and the soil sample is measured at certain intervals until the mass of the soil sample no longer changes. The change in diameter and the longitudinal height of the soil samples is recorded with a vernier caliper, and the corresponding mass moisture content is measured at the same time. The sample is then humidified after the dehumidification (drying) is completed, and the surface of the soil sample is uniformly humidified with misting droplets emitted by a water jet. In order to ensure an even water migration of the soil sample when absorbing water, a plastic wrap is used to seal the sample along the perimeter, and then the sealed sample is put into a sealed bag for secondary sealing. Finally, the sealed sample is placed into a constant temperature and constant humidity box for three days, and the changes of the sample are measured at regular intervals following the same procedure as that in the dehumidification (drying) process. To avoid the influences of inhomogeneous changes in the diameter and height of the soil sample on the final results, multiple measurements are conducted and the measured data are averaged for drawing the $\rho_{\mathrm{d}}-w$ curve, as shown in Figure 7.

As can be seen, the dry density of the specimen of $1.3 \mathrm{~g} /$ $\mathrm{cm}^{3}$ no longer changes (the volume of the specimen no longer changes) when the specimen is dehumidified to a moisture content of $18 \%$, i.e., the shrinkage limit of Hunan red clay is $18 \%$. In the process of dehumidification (drying) of the soil sample from the saturated state to the shrinkage limit, the large pore size in the soil is decreasing, resulting in the shrinkage of the soil volume, while the small pore size remains almost unchanged, so when the shrinkage limit is reached, the sample continues to dehumidify without volume change. Luan et al. [41] conducted an experimental study on the dry-wet cycles properties of Dalian remodeled clay, which showed that when the matric suction is increased to $633 \mathrm{kPa}$ (i.e., the shrinkage limit of Dalian clay), the increase in matric suction will have no significant effect on the shrinkage deformation of the specimen. In the hygroscopic (wetting) process of the specimen from the dry state of moisture absorption to the shrinkage limit, the sample's dry density did not change, indicating that the pore size has 

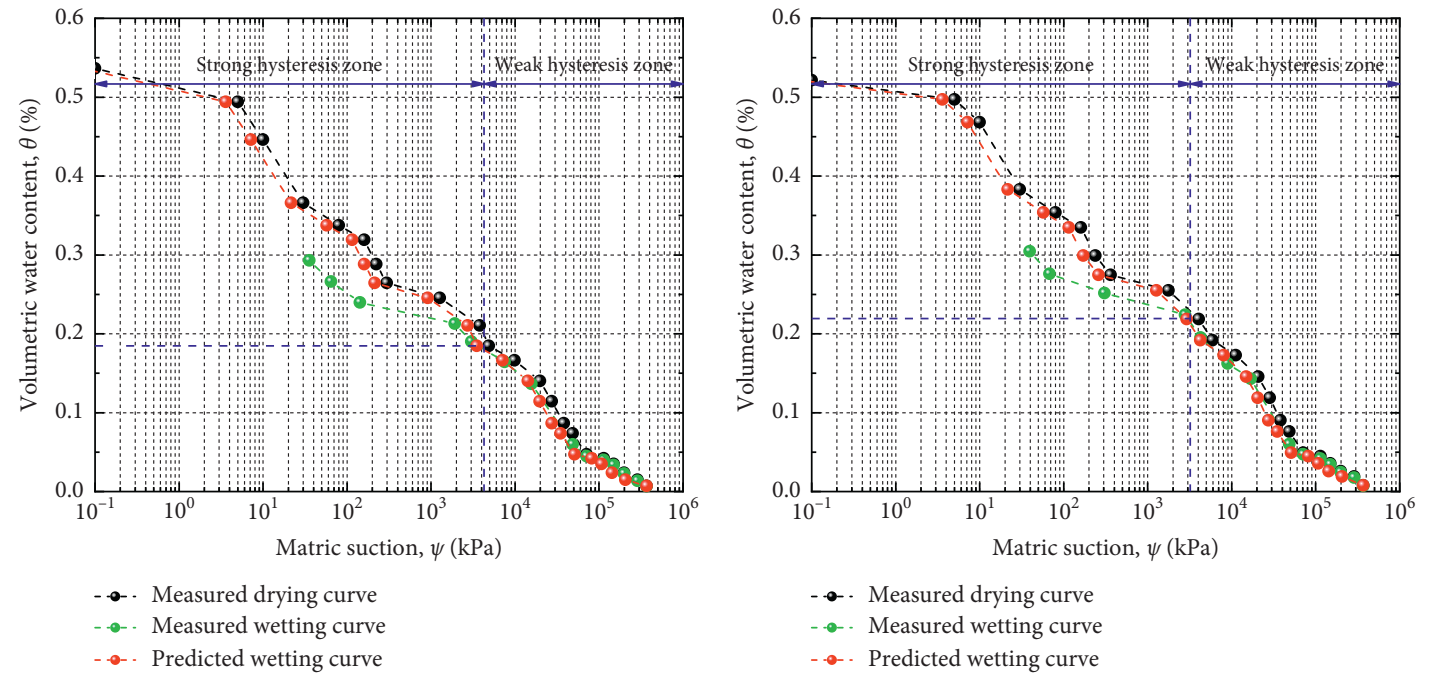

(a)

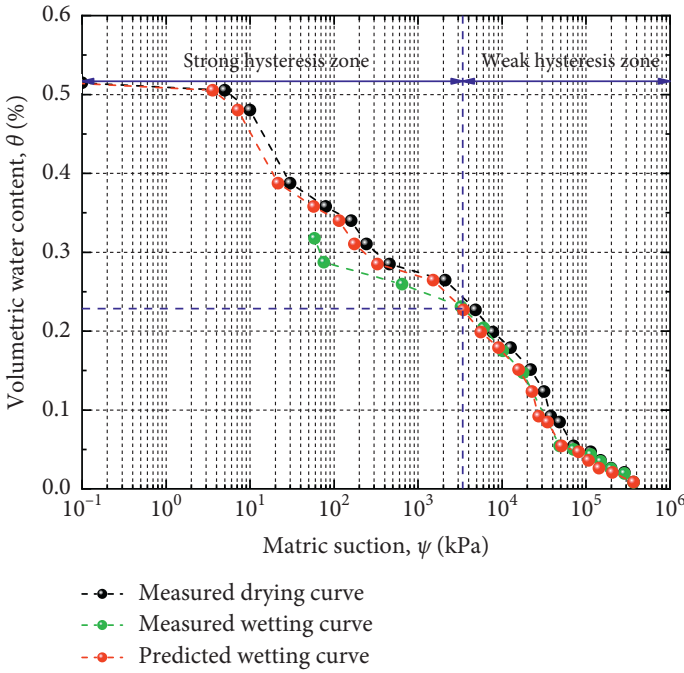

(b)

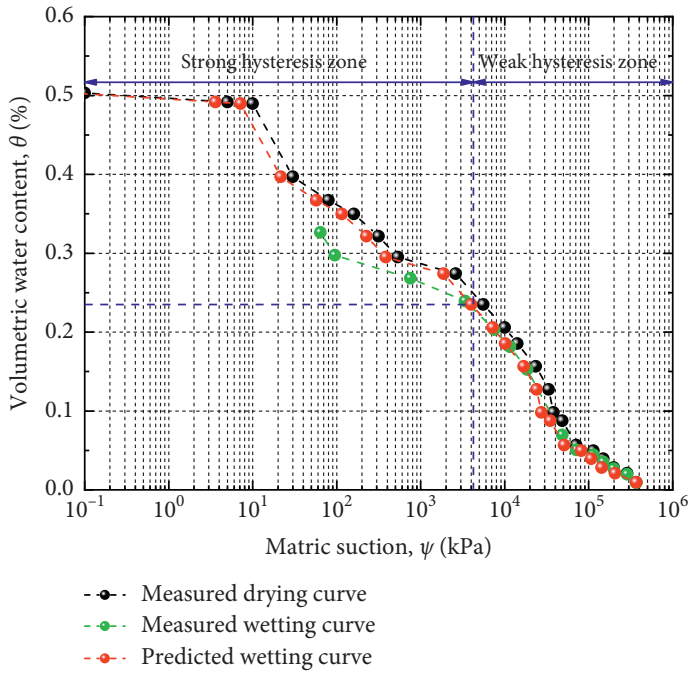

(c)

(d)
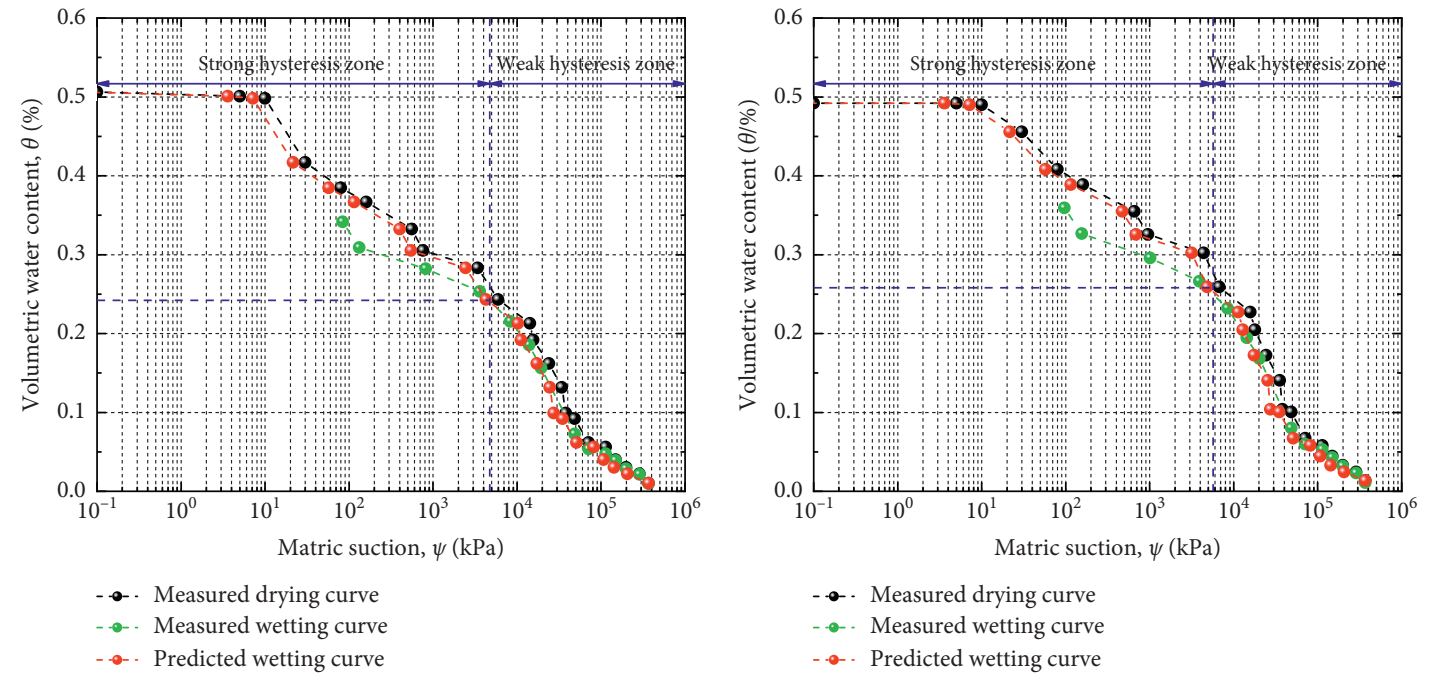

(e)

Figure 6: Continued. 


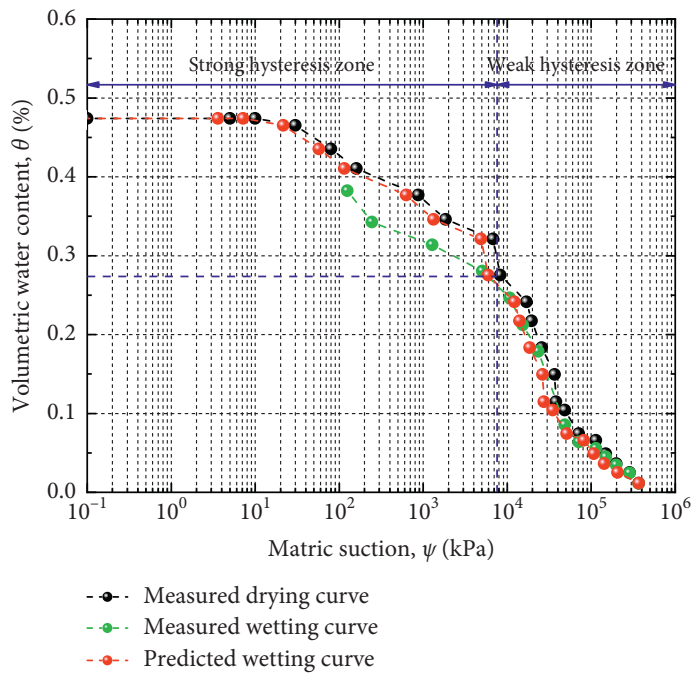

(g)

Figure 6: Wet and dry cycle predicted and measured curves: (a) $\rho_{\mathrm{d}}=1.3 \mathrm{~g} / \mathrm{cm}^{3}$; (b) $\rho_{\mathrm{d}}=1.35 \mathrm{~g} / \mathrm{cm}^{3} ;$ (c) $\rho_{\mathrm{d}}=1.4 \mathrm{~g} / \mathrm{cm}^{3} ;(\mathrm{d}) \rho_{\mathrm{d}}=1.45 \mathrm{~g} / \mathrm{cm}^{3}$; (e) $\rho_{\mathrm{d}}=1.5 \mathrm{~g} / \mathrm{cm}^{3}$; (f) $\rho_{\mathrm{d}}=1.6 \mathrm{~g} / \mathrm{cm}^{3} ;$ (g) $\rho_{\mathrm{d}}=1.7 \mathrm{~g} / \mathrm{cm}^{3}$.

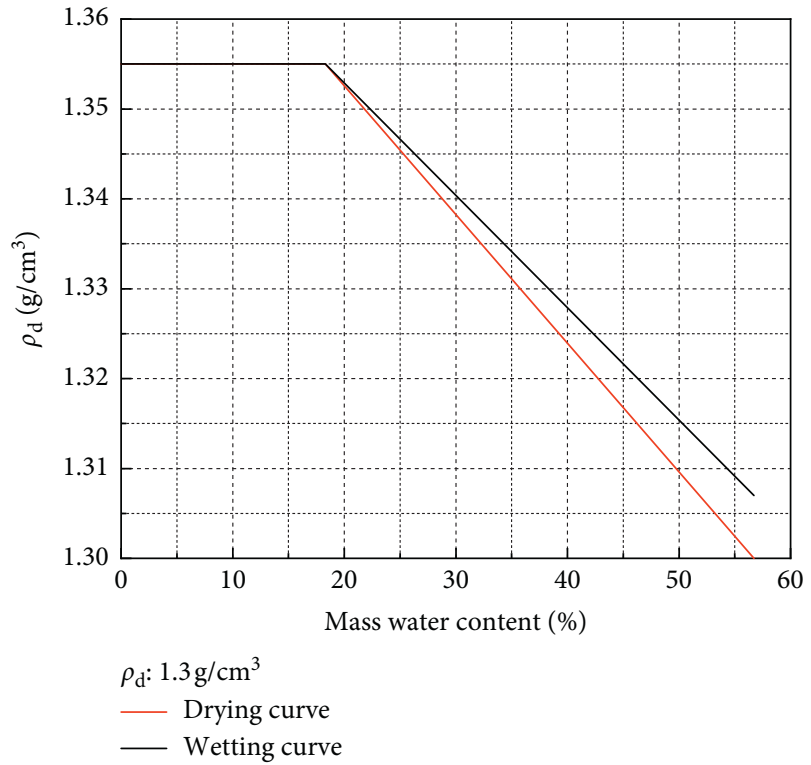

FIGURE 7: Wet and dry cycle soil volume change curve.

not changed. Moreover, the measured SWCC of the wetting process is below that of the drying process, as shown in Figure 6(a), which means that there is a weak hysteresis characteristic of the soil in the high suction range. For those pores with small internal dimensions, the sensitivity of soilwater characteristics is relatively low and the effects of contact angle on the hysteresis of the SWCC during dry-wet cycles are relatively weak, which, in combination with the predicted hygroscopic (wetting) curve, indicates that the contact angle changes during the dehumidification (drying) and hygroscopic (wetting) processes producing this phenomenon. Hence, it is reasonable to consider the effects of contact angle on the weak hysteresis zone corresponding to the high suction part of the SWCC during dry-wet cycles as the dominated influencing factor. The soil pore size becomes larger as the sample moves from shrinkage limit to saturated water content. Accordingly, the volume of the sample increases, but at this time the pore distribution characteristics differ greatly from the situation in the saturated state, so that the dry-wet cycles SWCC shows a significant hysteresis phenomenon [42]. Therefore, the hysteresis effect of dry-wet cycles soil-water characteristic curves generated in the low suction part is mainly influenced by the variation in pore size of the soil. In conclusion, the matric suction corresponding to the soil shrinkage limit is the boundary suction between the strong hysteresis zone and the weak hysteresis zone of the SWCCs.

\section{Conclusions}

In summary, the hysteresis effect of dry-wet cycling SWCCs in the full suction range is investigated by combination of the pressure plate method, the filter paper method, and the saturated salt solution method, and the hygroscopic (wetting) SWCCs are predicted by combining the contact angle hysteresis effect and the dehumidified SWCCs. Some conclusions are drawn as follows:

(1) During dry-wet cycles, there are distinct strong and weak hysteresis zones with a clear boundary between them in the SWCCs in the full suction range of seven kinds of Hunan red clay with different initial dry densities.

(2) By using the volume change curves during dry-wet cycles at the same initial dry density, it is found that the dominated influencing factor of the strong hysteresis zone is the pore size change inside the soil. When the number of large pores in the soil decreases, the strong hysteresis zone transforms to the weak hysteresis zone. Similarly, when the large pores 
gradually recover, the weak hysteresis zone can transform to the strong hysteresis zone.

(3) The overall effects of contact angle on the hysteresis effect of the SWCC in the full suction range are relatively weak. Considering smaller pores within the soil are less sensitive to soil-water characteristics, the contact angle hysteresis effect mainly has a significant influence on the weak hysteresis zone.

\section{Data Availability}

The data used to support the findings of this study are available from the corresponding author upon request.

\section{Conflicts of Interest}

The authors declare that they have no conflicts of interest regarding the publication of this paper.

\section{Acknowledgments}

The research was funded by the National Natural Science Foundation of China (grant number 51978249).

\section{References}

[1] U. D. Patil, A. J. Puppala, L. R. Hoyos, and A. Pedarla, "Modeling critical-state shear strength behavior of compacted silty sand via suction-controlled triaxial testing," Engineering Geology, vol. 231, pp. 21-33, 2017.

[2] T. Jin, X. Cai, Y. Chen, S. Jiang, and W. Wei, “A fractal-based model for soil water characteristic curve over entire range of water content," Capillarity, vol. 2, no. 4, pp. 66-75, 2019.

[3] W. R. Gardner, "Some steady-state solutions of the unsaturated moisture flow equation with application to evaporation from a water table," Soil Science, vol. 85, no. 4, pp. 228-232, 1958.

[4] R. H. Brooks and A. T. Corey, "Hydraulic properties of porous media," in Hydrology Papers, vol. 3, Colorado State University, Fort Collins, CO, USA, 1964.

[5] M. T. Van Genuchten, "A closed-form equation for predicting the hydraulic conductivity of unsaturated soils," Soil Science Society of America Journal, vol. 44, no. 5, pp. 892-898, 1980.

[6] D. G. Fredlund and A. Xing, "Equations for the soil-water characteristic curve," Canadian Geotechnical Journal, vol. 31, no. 4, pp. 521-532, 1994.

[7] P. S. Scott, "Hysteretic effects on net infiltration," Advances in Infiltration, vol. 11, pp. 163-170, 1983.

[8] J. R. Nimmo, "Semiempirical model of soil water hysteresis," Soil Science Society of America Journal, vol. 56, no. 6, pp. 1723-1730, 1992.

[9] M. Feng and D. G. Fredlund, "Hysteretic influence associated with thermal conductivity sensor measurements," Proceedings from Theory to the Practice of Unsaturated Regina, Sask, vol. 14, no. 2, pp. 14-20, 1999.

[10] A. Poulovassilis, "Hysteresis of pore water, an application of the concept of independent domains," Soil Science, vol. 93, no. 6, pp. 405-412, 1962.

[11] G. C. Topp, "Soil-water hysteresis measured in a sandy loam and compared with the hysteretic domain model," Soil Science Society of America Journal, vol. 33, no. 5, pp. 645-651, 1969.
[12] D. Flynn, H. McNamara, P. O’Kane, and A. Pokrovskii, Application of the Preisach Model in Soil-Moisture Hysteresis, NUI, Galway, Ireland, 2004.

[13] Y. Mualem, "Modified approach to capillary hysteresis based on a similarity hypothesis," Water Resources Research, vol. 9, no. 5, pp. 1324-1331, 1973.

[14] R. Haverkamp, P. Reggiani, P. J. Ross, and J.-Y. Parlange, "Soil water hysteresis prediction model based on theory and geometric scaling," Environmental Mechanics: Water, Mass and Energy Transfer in the Biosphere, vol. 129, pp. 213-246, 2002.

[15] X. S. Li, "Modelling of hysteresis response for arbitrary wetting/drying paths," Computers and Geotechnics, vol. 32, no. 2, pp. 133-137, 2005.

[16] C. F. Wei and M. M. Dewoolkar, "Formulation of capillary hysteresis with internal state variables," Water Resources Research, vol. 42, no. 7, 2006.

[17] C. G. Zhao, "Hysteresis model for soil-water characteristic curves," Chinese Journal of Geotechnical Engineering, vol. 30, no. 3, pp. 399-405, 2008 .

[18] X. D. Zhang, C. G. Zhao, Y. Liu, and G. Q. Cai, "Simulation and hysteresis model for soil-water characteristic curves," Journal of Engineering Geology, vol. 18, no. 6, pp. 920-925, 2010.

[19] J.-Y. Parlange, "Capillary hysteresis and the relationship between drying and wetting curves," Water Resources Research, vol. 12, no. 2, pp. 224-228, 1976.

[20] H. Q. Pham, D. G. Fredlund, and S. L. Barbour, "A study of hysteresis models for soil-water characteristic curves," $\mathrm{Ca}$ nadian Geotechnical Journal, vol. 42, no. 6, pp. 1548-1568, 2005.

[21] M. Nuth and L. Laloui, "Advances in modelling hysteretic water retention curve in deformable soils," Computers and Geotechnics, vol. 35, no. 6, pp. 835-844, 2008.

[22] Y. Mualem and A. Beriozkin, "General scaling rules of the hysteretic water retention function based on Mualem's domain theory," European Journal of Soil Science, vol. 60, no. 4, pp. 652-661, 2009.

[23] T.-K. Min and P. T. Huy, "A soil-water hysteresis model for unsaturated sands based on fuzzy set plasticity theory," KSCE Journal of Civil Engineering, vol. 14, no. 2, pp. 165-172, 2010.

[24] Y. Li, H. Luo, H. Li et al., "A brief review of dynamic capillarity effect and its characteristics in low permeability and tight reservoirs," Journal of Petroleum Science and Engineering, vol. 189, Article ID 106959, 2020.

[25] C. W. Ng and Y. W. Pang, "Experimental investigations of the soil-water characteristics of a volcanic soil," Canadian Geotechnical Journal, vol. 37, no. 6, pp. 1252-1264, 2000.

[26] W. He, M. H. Zhao, Y. G. Chen, and H. H. Wang, "Theoretical study of microscopical mechanisms and computational method of hysteresis in SWCCs," Rock and Soil Mechanics, vol. 31, no. 4, pp. 1078-1083, 2010.

[27] Z. Zhang, D. Zhou, X. G. Zhao, and L. Zhu, "Effects of initial density and drying-wetting cycle on soil water characteristic curve of unsaturated loess," Rock and Soil Mechanics, vol. 32, no. S2, pp. 132-136, 2011.

[28] J. Li, F. Y. Liu, L. Wang et al., "A research of affect factor on hysteresis behavior for soil-water characteristic curve," Journal of Hydraulic Engineering, vol. 46, no. S1, pp. 194-199, 2015.

[29] J. H. Wu and S. Yang, "Changes of matric suction in expansive soil under drying-wetting cycles using filter paper method," Transactions of the Chinese Society of Agricultural Engineering, vol. 33, no. 15, pp. 126-132, 2017. 
[30] D. A. Sun, J. R. Zhang, and H. B. Lv, "Soil-water characteristic curve of Nanyang expansive soil in full suction range," Rock and Soil Mechanics, vol. 34, no. 7, pp. 1839-1846, 2013.

[31] Y. Gao, D. A. Sun, J. R. Zhang, and T. Luo, "Soil-water characteristics of unsaturated soils considering initial void ratio and hydraulic path," Chinese Journal of Geotechnical Engineering, vol. 41, no. 12, pp. 2191-2196, 2019.

[32] G. Q. Cai, W. Liu, R. Z. Xu, J. Li, and C. G. Zhao, "Experimental investigation for soil-water characteristic curve of red clay in full suction range," Chinese Journal of Geotechnical Engineering, vol. 41, no. S2, pp. 13-16, 2019.

[33] F. Zheng, F. Y. Liu, and L. Wang, "Influence of unsaturated soil granularity on hysteretic behavior of soil-water characteristic curve," Journal of Water Resources and Architectural Engineering, vol. 17, no. 5, pp. 19-24, 2019.

[34] X. L. Zhu, "Research on the relationship between fractal characteristics of soil particle size and hydraulic properties for unsaturated soils," Hubei University of Technology, Master's thesis, 2019.

[35] B. W. Gong and C. G. Bao, "Measurement of matrix suction of expansive soil slope in Northern Hubei," Chinese Journal of Geotechnical Engineering, vol. 23, no. 1, pp. 64-67, 2001.

[36] F. Q. Bai, S. H. Liu, and J. Yuan, "Calibration test for total suction wetting curve of filter paper," Rock and Soil Mechanics, vol. 32, no. 8, pp. 2336-2340, 2011.

[37] U. D. Patil, L. R. Hoyos, and A. J. Puppala, "Characterization of compacted silty sand using a double-walled triaxial cell with fully automated relative-humidity control," Geotechnical Testing Journal, vol. 39, no. 5, pp. 742-756, 2016.

[38] D. A. Sun, W. J. Liu, and H. B. Lv, "Soil-water characteristic curve of Guilin lateritic clay," Rock and Soil Mechanics, vol. 35, no. 12, pp. 3345-3351, 2014.

[39] K. N. Manahiloh and C. L. Meehan, "Determining the soil water characteristic curve and interfacial contact angle from microstructural analysis of X-ray CT images," Journal of Geotechnical and Geoenvironmental Engineering, vol. 143, no. 8, Article ID 04017034, 2017.

[40] Z. Shen, Z. G. Zhao, and W. L. Kang, Colloid and Surface Chemistry, Chemical Industry Press, Beijing, China, 2012.

[41] M. T. Luan and Q. Yang, "Experimental study on behaviour of unsaturated remoulded clay subjected to drying and wetting cyclic paths," Chinese Journal of Rock Mechanics and Engineering, vol. 26, no. 9, pp. 1862-1867, 2007.

[42] G. L. Tao, J. R. Zhang, X. S. Zhuang, and L. Yang, "Influence of compression deformation on the soil-water characteristic curve and its simplified representation method," Journal of Hydraulic Engineering, vol. 45, no. 10, pp. 1239-1246, 2014. 